\title{
Percutaneous Intramyocardial Delivery of Mesenchymal Stem Cells Induces Superior Improvement in Regional Left Ventricular Function Compared with Bone Marrow Mononuclear Cells in Porcine Myocardial Infarcted Heart
}

\author{
Bo Tao ${ }^{*}$, Mingliang Cui ${ }^{*}$, Chen Wang ${ }^{*}$, Sai Ma1 ${ }^{1}$ Feng Wu${ }^{1}$, Fu Yi1 ${ }^{1}$, Xing Qin ${ }^{1}$, Junting Liu ${ }^{4}$, Haichang

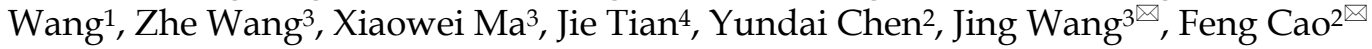 \\ 1. Department of Cardiology, Xijing Hospital, Fourth Military Medical University, Xi' an 710032, China; \\ 2. Department of Cardiology, Chinese PLA General Hospital,Beijing 100853, China; \\ 3. Department of Nuclear Medicine,Xijing Hospital, Fourth Military Medical University, Xi'an 710032, China; \\ 4. School of Life Science and Technology, Xidian University, Xi'an 710126, China. \\ * Contributed equally to the study.
}

$\triangle$ Corresponding author: Feng Cao, MD. PhD. Department of Cardiology, Chinese PLA General Hospital, Beijing 100853, China; Tel: +86-10-55493168; Fax: +86-29-84773464; E-mail:wind8828@gmail.com or Jing Wang, MD. PhD. Department of Nuclear Medicine, Xijing Hospital, Fourth Military Medical University, Xi' an 710032, China; E-mail: wanging@fmmu.edu.cn.

(c) Ivyspring International Publisher. This is an open-access article distributed under the terms of the Creative Commons License (http://creativecommons.org/ licenses/by-nc-nd/3.0/). Reproduction is permitted for personal, noncommercial use, provided that the article is in whole, unmodified, and properly cited.

Received: 2013.10.25; Accepted: 2014.10.02; Published: 2015.01.01

\begin{abstract}
Aim: To investigate the efficacy and feasibility of percutaneous intramyocardial injection of bone marrow mesenchymal stem cells (MSC) and autologous bone marrow-derived mononuclear cells (BMMNC) on cardiac functional improvement in porcine myocardial infarcted hearts. Methods and Results: Acute myocardial infarction (AMI) was induced in 22 minipigs by temporary balloon occlusion of the left anterior descending coronary artery for $60 \mathrm{~min}$. Two weeks post AMI, BMMNC $\left(n=7,245 \pm 98 \times 10^{6}\right)$, MSC $\left(n=8,56 \pm 17 \times 10^{6}\right)$, or phosphate buffered saline (PBS; $n=$ 7) were injected intramyocardially. Cardiac function and myocardial perfusion were analyzed by echocardiography and gated single-photon emission computed tomography/computed tomography (SPECT/CT) at I week before AMI and 2 and 10 weeks after AMI. Cell engraftment, proliferation, vascular density, and cardiac fibrosis were evaluated by histology analysis. In all groups, the echocardiography revealed no significant change in the left ventricular ejection fraction (LVEF), left ventricular end-systolic volume (LVESV), or left ventricular end-diastolic volume (LVEDV) at 10 weeks after AMI compared with those at 2 weeks after AMI. However, the wall motion score index (WMSI) and left ventricular systolic wall thickening (WT\%) were significantly improved at 10 weeks compared with those at 2 weeks after AMI in the MSC group (WMSI I.55 \pm 0.06 vs. $1.87 \pm$ 0.10 , WT $33.4 \pm 2.3 \%$ vs. $24.8 \pm 2.7 \%, p<0.05$ ) but not in the BMMNC group. In addition, myocardial perfusion quantified by SPECT/CT was improved in both the MSC and BMMNC groups, whereas the MSC group showed a superior improvement in vascular density and collagen volume fraction $(p<0.05)$. Conclusion: This preclinically relevant study suggests that when delivered by percutaneous (transcatheter) intramyocardial injection, MSC might be more effective than BMMNC to improve ischemia and reperfusion after AMI.
\end{abstract}

Key words: Angiogenesis, Imaging, Myocardial infarction, Remodeling, Stem cells. 


\section{Introduction}

Ischemic heart disease is a leading cause of human deaths worldwide. Acute and chronic ischemic injury leads to a permanent loss of cardiac tissue and ultimately heart failure. Recently, cell-based therapy has emerged as a novel potential approach for regenerating heart tissue. Several cell types have been shown to improve heart functional recovery after ischemic injury. Stem cell therapy in patients with acute myocardial infarction (AMI) has shown mixed results [1], and the wide diversity of technical procedures used in each study has made comparison difficult. Among various cell types, bone marrow-derived mononuclear cells (BMMNC) have been most widely applied in clinical trials and basic research [2-7]. Bone marrow-derived mesenchymal stem cells (MSC) have also emerged as a promising cell population, which could be used as allogeneic "off-the-shelf" cell products because of the multilineage potential and immuno-privileged properties of these cells [8-13]. However, the best cell type for the treatment of myocardial infarction remains uncertain, and the mechanisms underlying cellular therapy are not well understood. Among several delivery methods for cellular therapy, intramyocardial delivery seems to be preferable to intravenous and intracoronary deliveries [14]. The safety of intramyocardial delivery has also been demonstrated in a clinical trial [15]. The present study aimed to compare functional and structural recoveries after AMI in minipigs treated with percutaneous intramyocardial injection of autologous BMMNC and MSC.

\section{Materials and Methods}

\section{Study design}

The summary of the study design is shown in Figure 1. In minipigs, AMI was induced by balloon occlusion of the left anterior descending (LAD) artery for $60 \mathrm{~min}$, followed by reperfusion. Two weeks after AMI, the minipigs were randomly assigned to blinded percutaneous intramyocardial delivery of phosphate buffered saline (PBS) as a control medium (CON), autologous BMMNC, or MSC. Echocardiography and gated single-photon emission computed tomography/computed tomography (SPECT/CT) were performed 1 week before AMI and 2 and 10 weeks after AMI. All animal procedures were conducted in conformity with the National Institutes of Health Guide for the Care and Use of Laboratory Animals, and all experiments were performed in accordance with the Helsinki Declaration. The experimental protocol was approved by the Fourth Military Medical University Committee on Animal Care.

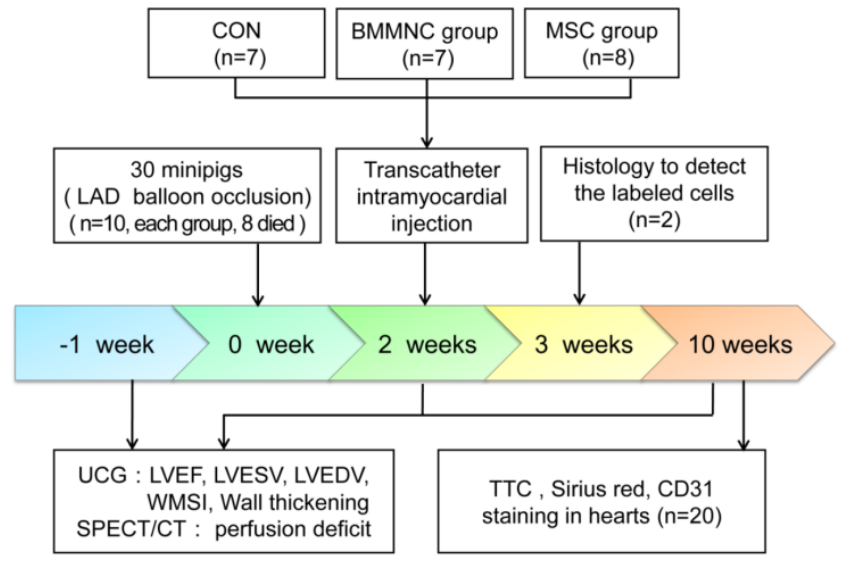

Figure I. Schematic representation of the study timeline.

\section{Cell preparation}

Porcine BMMNC were aspirated from bilateral iliac crests and isolated by density gradient centrifugation following standard protocols [7]. MSC were obtained from mononuclear cells. Briefly, the mononuclear cells were seeded into culture flasks and amplified in adherent cell cultures. Cells were seeded at 2 $\times 10^{5} / \mathrm{cm}^{2}$ in DMEM/F12 (Hyclone, USA) containing $20 \%$ fetal bovine serum (FBS) (Gibco, BRS, USA). Cultures were expanded to passage 5 .

To characterize MSC, the cultured cells were differentiated along adipogenic, osteogenic, and chondrogenic lineages as previously described [8] and analyzed by flow cytometry for MSC markers. For flowcytometric analysis, the cells were stained with human antibodies against CD34, CD44, CD45, and CD90, which were conjugated with fluorescein isothiocyanate (FITC) or phycoerythrin (PE) (Abcam, Cambridge, MA, USA).

MSC were genetically transfected with lentiviral vectors encoding green fluorescent protein (GFP) (Genechem, Shanghai, China). The multiplicity of infection (MOI) was 20. The quantification of GFP transduction efficiency in MSC was performed following previously described protocols [16]. The cells were then frozen in liquid nitrogen until required for the next experiment. Before injection, MSC were rapidly thawed and washed to remove DMSO, then resuspended in PBS. Immediately before injection, the viability of all thawed MSC lots was verified to be $>85 \%$ using trypan blue dye exclusion. BMMNC were labeled with CM-DiI following the manufacturer's instructions (Molecular Probes, Invitrogen, USA).

\section{Inductionof porcine myocardial infarction}

Chinese experimental minipigs $(35 \pm 5 \mathrm{~kg}$; China Agricultural University, Beijing, China) were anesthetized with ketamine hydrochloride $(20 \mathrm{mg} / \mathrm{kg}$, IM; 
Beijing Chemical Reagent Company, Beijing, China) followed by a continuous infusion of propofol $(0.15$ $\mathrm{mg} / \mathrm{kg} / \mathrm{min}, \mathrm{IV}$ ) and were incubated and ventilated with a 1:1 mixture of air and oxygen. After the incision of the skin, a $6 \mathrm{~F}$ arterial sheath was placed in the right or left femoral artery by percutaneous puncture or direct vision puncture. Femoral artery angiography was performed (Figure 2A) and heparin (150IU/kg) was administered to obtain a 2.5-fold increase in activated coagulation time. After the injection of heparin, a $6 \mathrm{~F}$ Judkins right (JR) 4.0 coronary artery guiding catheter was engaged into the opening of the coronary artery. Selective coronary angiography was performed, and the target blocking location was determined. Myocardial infarction was induced by the temporary balloon occlusion of the distal LAD coronary artery below the second diagonal branch (Figure 2B-C) for $60 \mathrm{~min}$, followed by reperfusion with an inflated angioplasty balloon as previously described [14]. Twelve-lead electrocardiograms (ECGs) were recorded at $25 \mathrm{~mm} / \mathrm{s}$ and $10 \mathrm{~mm} / \mathrm{mV}$ in anesthetized and immobilized minipigs in the supine position.
Precordial leads were included to precisely determine the ventricular wall segment affected (Figure 2E). Continuous ECG monitoring was performed to warn of arrhythmia. Lidocaine $(0.015-0.03 \mathrm{mg} / \mathrm{kg} / \mathrm{min})$ was intravenously infused throughout the procedure. After the procedure, all the animals received antibiotics (amoxicillin $7 \mathrm{mg} / \mathrm{kg} / 24 \mathrm{~h}$ ) for 5 days.

\section{Cell delivery}

For medium delivery or cell transplantation, a percutaneous transcatheter approach was established through the femoral artery. Intramyocardial delivery was performed with an injection catheter system (kindly provided by Microport Corp., Shanghai, China) (Figure 2F-H). Injection sites were confirmed by contrast injection before cell delivery (Figure 2D). Multiple injections (an average of 15 per time point, $0.25-0.3 \mathrm{ml}$ per injection) within the hypokinetic and akinetic zones were performed under simultaneous fluoroscopy C-arm and echocardiographic guidance in each animal heart.
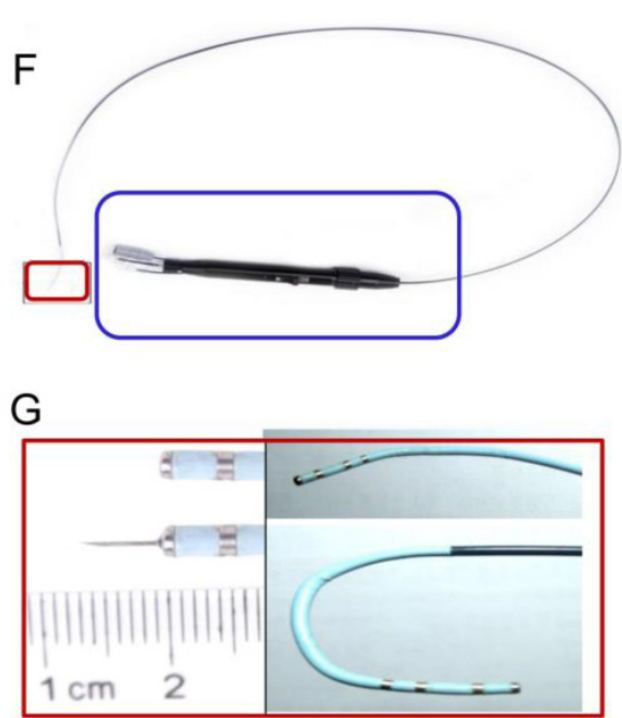

$\mathrm{H}$

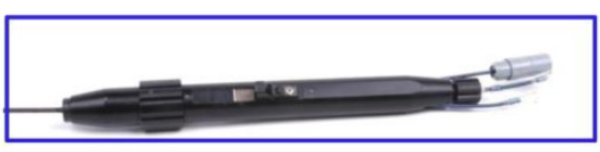

Figure 2. (A) Minipig femoral artery angiogram. (B) Coronary angiography to determine the location of the balloon occluded left anterior descending (LAD) coronary artery. (C) Blood flow was completely blocked after balloon occlusion of the distal LAD coronary artery, but blood flow in the second diagonal branch is clearly seen. (D) Injection of contrast medium to confirm the location of the needle prior to the injection of bone marrow-derived mononuclear cells (BMMNC) or mesenchymal stem cells (MSC). (E) I2-lead electrocardiogram confirmed ST segment elevation in anterior wall leads $30 \mathrm{~min}$ after balloon occlusion. (F) Intramyocardial injection catheter system. An integrated device with a system $(\mathrm{H})$ to control needle movement from a withdrawn to an extended position and to control tip movement from a straight to a deflected position (G). 


\section{Echocardiographic evaluation of left ventricu- lar function}

Two-dimensional (2D) and M-mode echocardiography were performed with a VIVID 7 ultrasound imaging system (GE, New York, USA) 1 week before AMI, and 2 and 10 weeks after AMI. The echocardiography results 1 week before AMI were taken as the baseline. Cardiac apex ultrasound imaging by transthoracic echocardiography is difficult in pigs. Therefore, images were obtained only in the parasternal short axis view (at apical, papillary, and mitral valve levels). All measurements were performed in triplicate by two technicians blinded to the study and were averaged to ensure quality.

Left ventricular ejection fraction (LVEF), end-systolic volume (LVESV), and end-diastolic volumes (LVEDV) were determined in the parasternal short axis as previously described [17]. The wall motion score index (WMSI) was calculated by ranking wall motion as normal (1), mildly hypokinetic (2), severely hypokinetic (2.5), akinetic (3), or dyskinetic (4) and then by dividing the sum of the wall motion score with the number of visualized segments. Regional wall thickness was measured at end-diastole (DWT) and end-systole (SWT) individually on2D echocardiograms. LDWT and SWT were measured, and left ventricular systolic wall thickening (WT\%) was calculated as WT $\%=($ SWT - DWT $) / D W T \times 100 \%$.

All images were recorded and analyzed offline and results were determined by the consensus of two experienced ultrasound technicians blinded to the treatment assignment.

\section{Quantitative gated SPECT/CT analysis}

ECG-gated SPECT/CT imaging was performed 1 week before AMI and 2 and 10 weeks after AMI with a commercially available system (SymbiaT2, Siemens).The myocardial perfusion images acquired1 week before AMI were taken as baseline data. The animals were sedated with an intravenous injection of ketamine and were placed in the right lateral decubitus position. Imaging was assessed using a dose of 0.3 $\mathrm{mCi} / \mathrm{kg}$ 99mTc-sestamibi (99mTc-MIBI) injected into the ear vein. CT images were acquired with the 2-sliceCT component of the SPECT/CT scanner. Myocardial perfusion images were acquired $60 \mathrm{~min}$ after 99mTc-MIBI injection using a dual-head camera with high-resolution collimators. The camera energy window $(20 \%)$ was set to the $140 \mathrm{keV}$ photo peak of $99 \mathrm{mTc}-\mathrm{MIBI}$. Thirty-two images $(64 \times 64$ matrix $)$ were acquired for $30 \mathrm{~s}$ each, with a $180^{\circ}$ rotation. Tomograms were reconstructed in the vertical and horizontal long-axis and short-axis planes. Quantitative-gated SPECT software (QGS; Cedars Cardiac Quantification) was used for the processing of all re- cordings and for the semi-quantitative assessment of left ventricular volumes and perfusion defects. The fusion of SPECT and CT images was performed on a processing station (Syngo MI VA30A; Siemens, Germany). Two experienced nuclear medicine technicians who were blinded to the treatment allocation processed all the recordings.

\section{Histological analysis}

Three weeks after AMI, two minipigs in the cell transplantation group were sacrificed for the observation of cell survival. The animals were anesthetized with ketamine hydrochloride and were humanely sacrificed with an intravenous injection of saturated potassium chloride solution. Cardiac tissues were harvested, snap-frozen in nitrogen-cooled isopentane, and $6-\mu \mathrm{m}$ serial sections were prepared. Fluorescence microscopy (Olympus BX51; Olympus, Japan) was performed to detect labeled MSC and BMMNC. Ten weeks after AMI, all minipigs were sacrificed, and their tissues were harvested. The labeled cells in the cell transplantation group were detected following the steps described above.

The capillary density was determined in the peri-infarct zone. The sections were stained using anti-CD31 antibody (mouse monoclonal; Abcam). Investigators blinded to the treatment allocation examined the sections to quantify the positively stained vessels. The capillaries were counted in five randomly chosen high-power fields (HPFs) in every section. The results were expressed as capillaries per HPF. In addition, longitudinal myocardial sections were stained for 10 min with 1\% 2,3,5-triphenyltetrazoliumchloride (TTC) to delineate infarct borders. The collagen content was determined in picric acid/Sirius red-stained slides as described previously [18], measured with an interactive computerized image analysis system (Optimas 5.2 color image analysis; Germany), and expressed as a percentage of the area of the field occupied by collagen (collagen volume fraction; CVF). The mean of 15 fields captured with a $\times 20$ objective was calculated. In this process, we blindly investigated the presence of Type I and Type III collagen with circularly polarized light on randomly selected picric ac$\mathrm{id} /$ Sirius red-stained sections from the infarct border zone of hearts from the CON, BMMNC, and MSC groups.

\section{Statistical analysis}

Continuous variables that approximated the normal distribution are presented as mean \pm standard error of the mean (SEM). The characteristics of the minipigs were evaluated using the Kruskal-Wallis test. Multiple group comparisons were performed by one-way analysis of variance (ANOVA) followed by 
the least significant difference (LSD) $t$-test for post hoc analysis. Comparisons between the two independent groups were analyzed using the Student's $t$-test. Two-sided tests were used throughout, and $p$-values $<0.05$ were considered statistically significant. SPSS software package version 14.0(SPSS, Chicago, IL, USA) was used for data analysis.

\section{Results}

\section{Animal survival during the experimental pro- cedure}

In total, 30 animals underwent the infarct procedure. During the procedure, six animals died as a result of intractable ventricular fibrillation and two died from cardiac arrest. Therefore, 22 pigs were included in the study and were divided into three groups. The mean number of cells implanted was $245 \times 10^{6}$ for the BMMNC group and $56 \times 10^{6}$ for the MSC group. There were no deaths resulting from the injection of cells or medium.

\section{Cell characterization}

As shown in Figure 3, the flowcytometry of MSC revealed the uniform expression of CD44 and CD90 but not of CD34 or CD45. After genetic labeling and multiple passages, the pluripotency of MSC was confirmed by the capacity to differentiate into adipogenic, osteogenic, and chondrogenic lineages in vitro.

\section{Regional wall motion was improved in the MSC group}

Echocardiography was performed to monitor global and regional cardiac functions. Baseline functional values were equivalent in the three groups. After AMI, there was a significant reduction in LVEF, which did not differ among the three groups. At week 10, there was no significant improvement in cardiac volume or global function in the three groups. Although LVEF increased in animals treated with BMMNC or MSC, the change was not significant $(p>$ 0.05). Neither LVESV nor LVEDV was significantly improved in any of the three groups (Figure $4 \mathrm{~A}-\mathrm{C}$ ).

To assess regional wall motion, the left ventricular WMSI and systolic WT\% were calculated. Two weeks after AMI, the WMSI was significantly increased and systolic WT\% was significantly reduced in all three groups compared with baseline (WMSI: $1.00 \pm 0.10$ to $1.80 \pm 0.14$, WT\%: $39.3 \pm 2.0 \%$ to $24.5 \pm$ $2.3 \%, p<0.01, \mathrm{n}=22$; Figure $4 \mathrm{D}-\mathrm{F})$. Ten weeks after AMI, WMSI and systolic WT\% were significantly improved only in animals that received MSC (WMSI: $1.87 \pm 0.10$ to $1.55 \pm 0.06$, WT $\%: 24.8 \pm 2.7 \%$ to $33.4 \pm$ $2.3 \%, p<0.05$; Figure $4 \mathrm{D}-\mathrm{F})$. Therefore, the regional left ventricular function was better preserved in the MSC group compared with the BMMNC and CON groups.
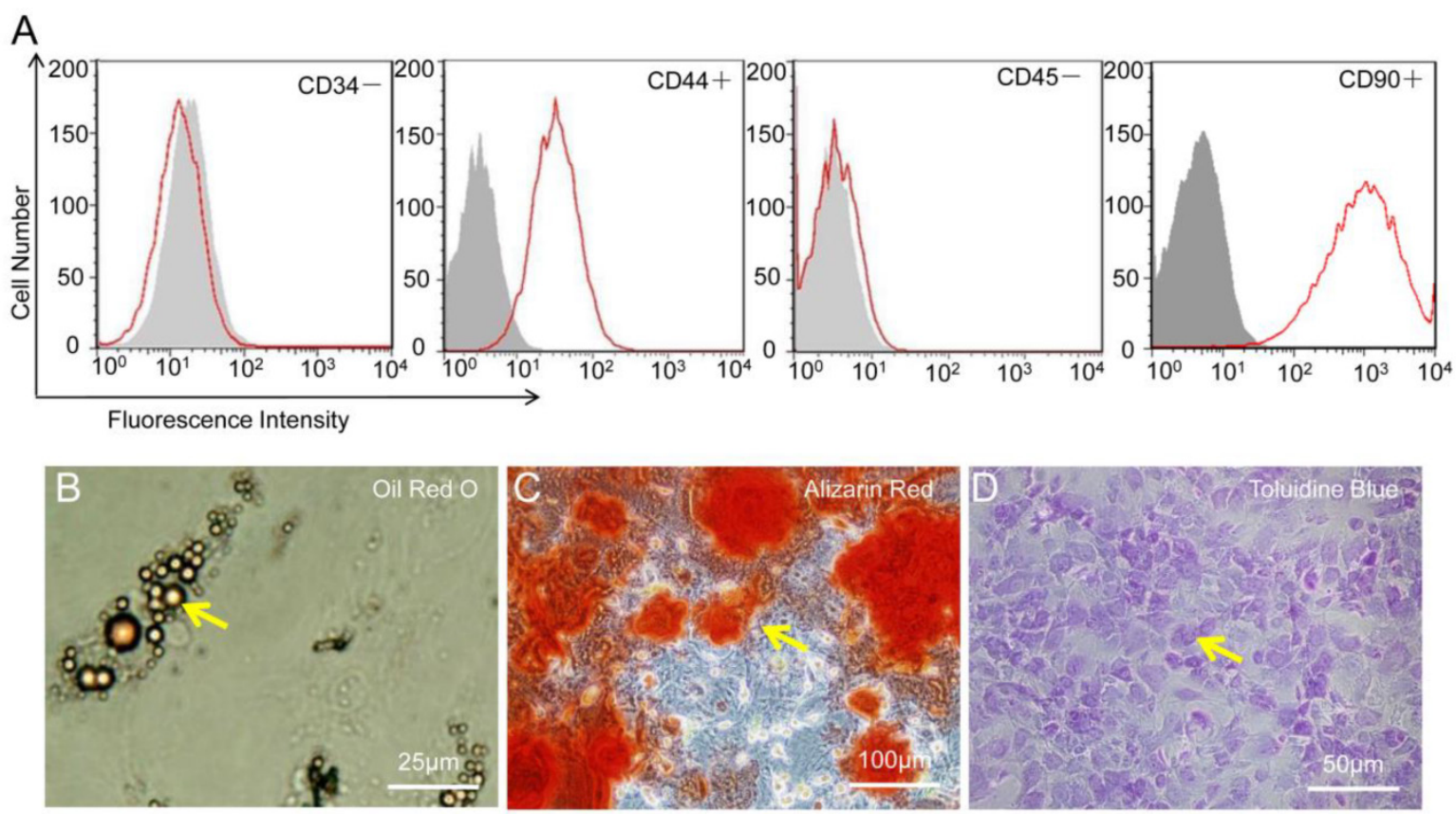

Figure 3. Characterization of mesenchymal stem cells (MSC). (A) MSC were positive for CD44 and CD90 but negative for CD34 and CD45. (B-D) MSC show potential for differentiation towards adipogenic (Oil Red O staining), osteogenic (Alizarin Red), and chondrogenic (Toluidine Blue) lineages. 

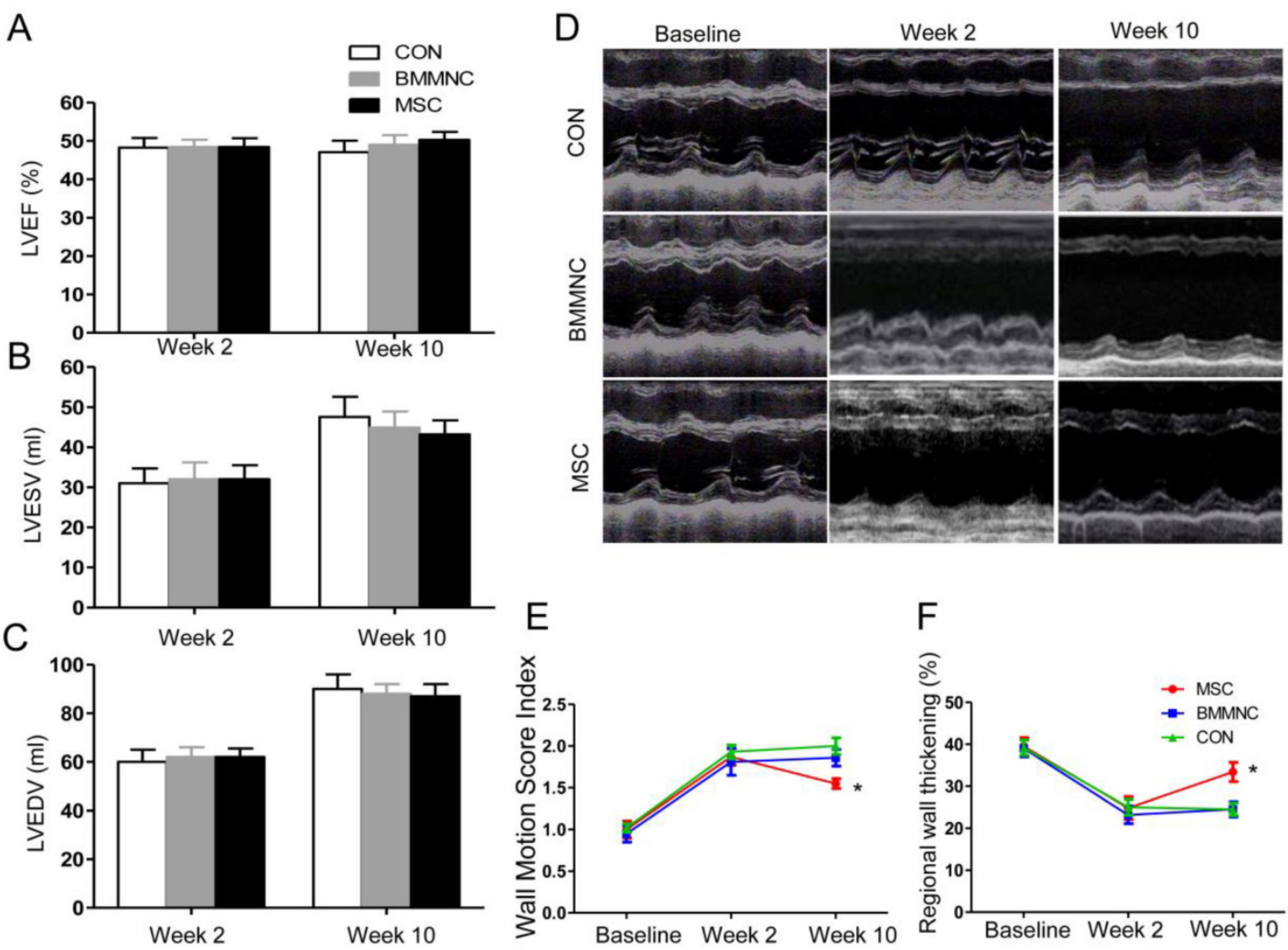

Figure 4. (A-C) Echocardiographic evaluation of cardiac function. LVEF, LVESV, and LVEDV were not significantly improved in the three groups. (D) Echocardiographic evaluation of regional cardiac contractility. Representative M-mode echocardiographic data for minipigs receiving medium (CON), BMMNC, or MSC I week before AMI and 2 and 10 weeks after AMI. Wall motion score index $(E)$ and systolic wall thickening $\%(F)$ were significantly improved only in the MSC group. $\left({ }^{*} p<0.05\right)$.

\section{Myocardial perfusion was improved by cellular therapy in both BMMNC and MSC groups}

Two weeks after AMI, all the experimental animals showed a significant perfusion defect in the anteroapical and distal septal walls of the myocardium as assessed by SPECT/CT (Figure 5A). Ten weeks after AMI, the animals treated with BMMNC or MSC showed a significant reduction in the total perfusion deficit (TPD) $(27.5 \pm 1.0 \%$ to $23.4 \pm 1.5 \%$ in the BMMNC group, $28.4 \pm 1.8 \%$ to $23.2 \pm 1.4 \%$ in the MSC group, $p<0.05)$. The animals injected with the medium alone did not show any reduction in TPD $(29.2 \pm$ $1.4 \%$ to $30.4 \pm 1.2 \%, p>0.05$ ), as defined by semi-quantitative SPECT analysis (Figure 5B-D). Absolute change in perfusion deficit was significantly larger in the BMMNC and MSC groups compared with the CON group $(-4.1 \pm 1.3 \%$ in the BMMNC group, $-5.2 \pm 1.6 \%$ in the MSC group vs. $1.2 \pm 0.35 \%$ in the CON group, $p<0.05$, Figure $5 \mathrm{E}$ ). Furthermore, the absolute change in perfusion deficit was larger in the MSC group than in the BMMNC group, although the difference was not significant. Echocardiography analysis results for LVEF, LVESV, and LVEDV showed similar trends, but no significant change was found (data not shown).

\section{Capillary density and fibrosis were better im- proved in the MSC group than the BMMNC group}

Infarct patterns were shown on representative tetrazolium (TTC) stains from the three groups (Figure 6A-C). Sirius red staining (Figure 6D-F) and polarized light microscopy of the infarct border zone (Figure 6G-I) showed decreased fibrosis (49.8 $\pm 2.4 \%$ in the MSC group vs. $56.8 \pm 1.9 \%$ in the BMMNC group and $69.2 \pm 3.6 \%$ in the CON group, Figure $6 \mathrm{~J}$ ) and scar tissue was better organized in the MSC group compared with the CON and BMMNC groups. 
A

C
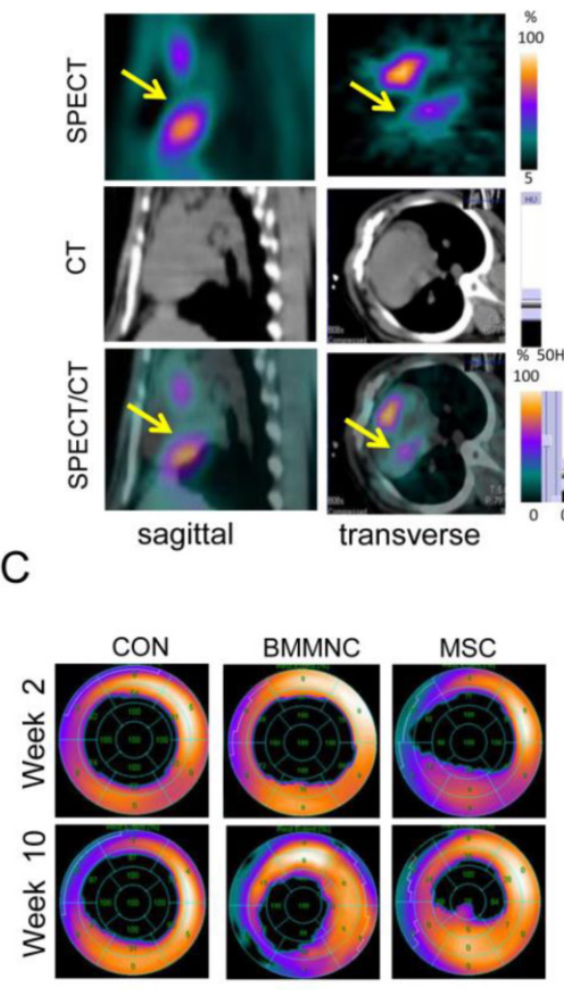

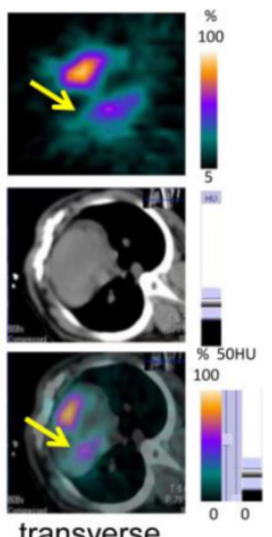

transverse
B

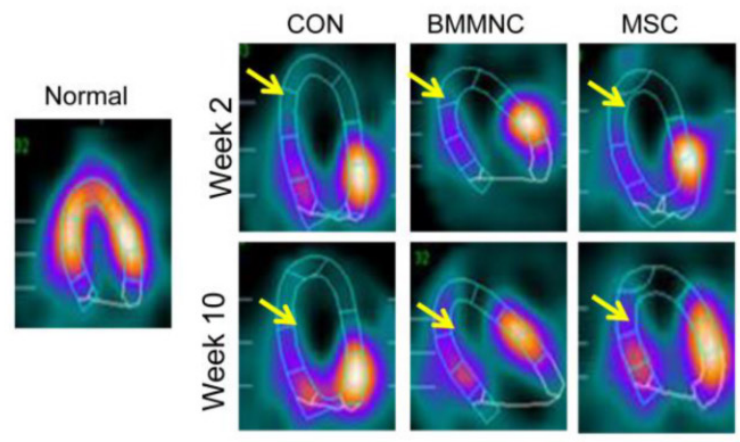

E

$B$
D

D

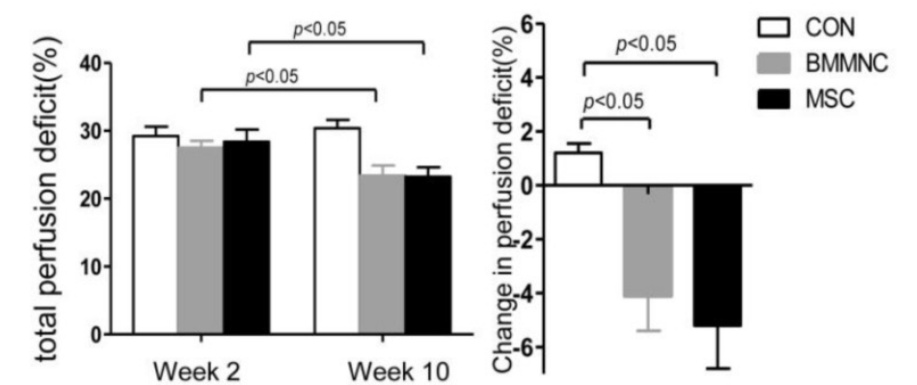

Figure 5. (A) Hybrid imaging of myocardial perfusion SPECT and CT. All groups showed a significant perfusion defect in the anteroapical and distal septal wall of the myocardium. (B) Representative horizontal long axis slices and (C) polar bull's eye plot at 2 and 10 weeks after AMI showed a significant reduction in myocardial perfusion in the BMMNC and MSC groups. (D-F) Semi-quantitative assessment of total perfusion deficit and absolute change in perfusion deficit showed a significant reduction in the BMMNC and MSC groups.
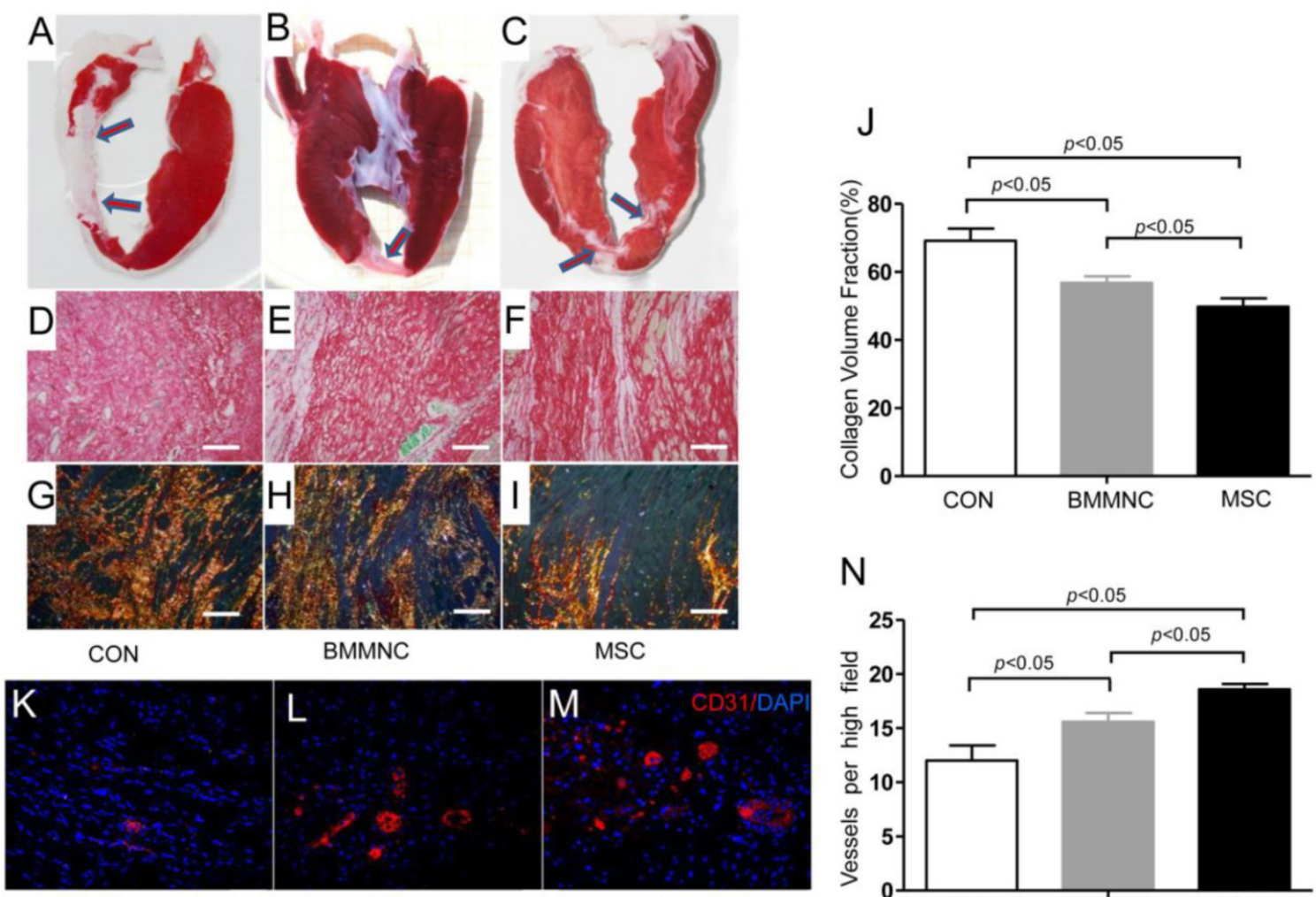

CON

BMMNC

MSC

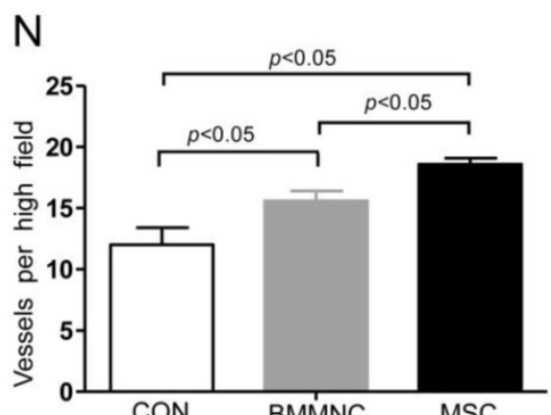

Figure 6. Infarct borders were identified in representative samples from the CON, BMMNC, and MSC groups by TTC stains (A-C, arrowheads). After Sirius red staining (D-F), polarized light microscopy $(\mathrm{G}-\mathrm{I})$ revealed a better-organized fibrous scar in the MSC group (I) compared with the CON group (G) and the BMMNC group (H). Scale bar $=50 \mu \mathrm{m}$. The area occupied by the collagen volume fraction (CVF) was assessed by Sirius red staining in the infarcted tissue (J).Vascular density was determined in CD3I stained sections from the border zone. The overall number of $C D 3 I+$ vessels was significantly greater in the MSC treated hearts than in BMMNC and CON hearts. Scale bar $=50 \mu m(K-N)$. 
A
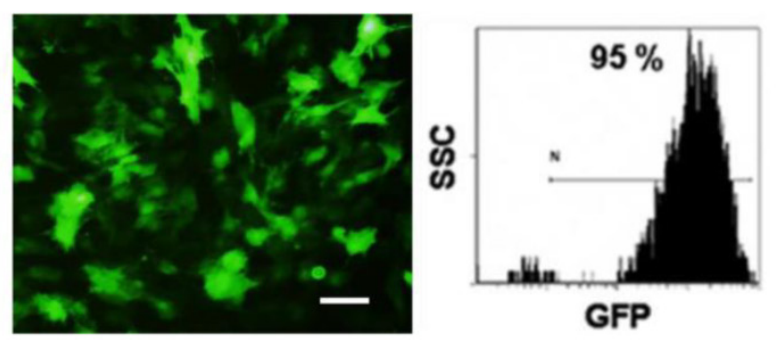

MSCGFP+

B
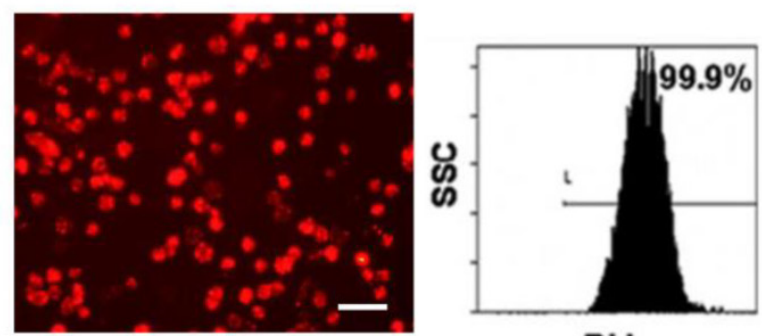

Di I

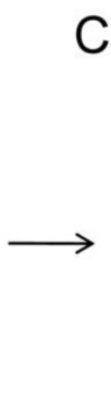

C

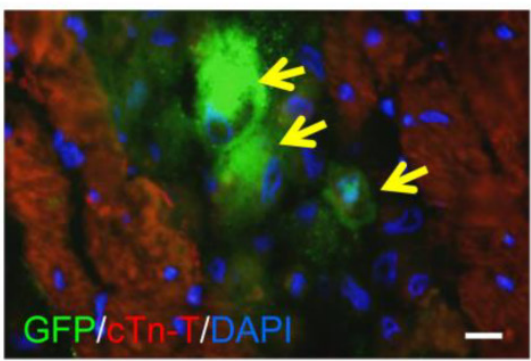

D

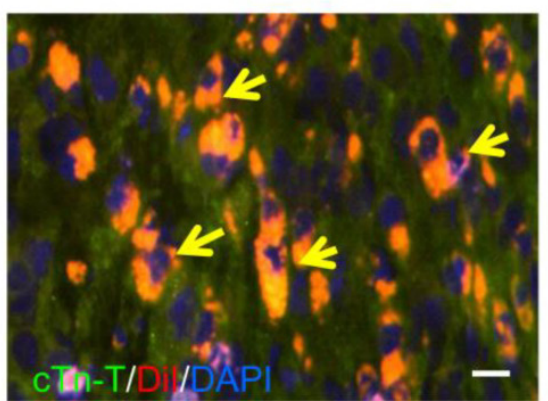

BMMNC ${ }^{\text {Dilt }}$

Figure 7. Immunofluorescence staining for cell survival in heart tissue 3 and 10 weeks after AMI. GFP labeled MSC (A) and Dil labeled BMMNC(B) could be detected 3 weeks after AMI(C-D) but not 10 weeks after AMI (yellow arrows).Scale bar $=50 \mu \mathrm{m}(A-B)$; scale bar $=25 \mu \mathrm{m}(C-D)$.

To examine whether changes in regional function and perfusion were associated with enhanced neovascularization, vascular density was determined on CD31-stained sections from the border zone. Ten weeks after AMI, immunofluorescence staining for CD31 antibody indicated significant angiogenesis in cell-treated hearts with more CD31-expressing capillaries present in peri-infarct regions of cell-treated hearts compared with medium-treated hearts. The semi-quantitative evaluation of CD31-positive capillary numbers per high-power field indicated that vascular density was significantly greater in the cell-treated groups than in the medium-treated group $(18.6 \pm 0.5$ in the MSC group and $15.6 \pm 0.8$ in the BMMNC group vs. $12 \pm 1.4$ in the CON group, Figure $6 \mathrm{~K}-\mathrm{N})$.

\section{Labeled cells were detected three weeks after AMI}

CM-DiI-labeled BMMNC and GFP-labeled MSC were found in the cardiac tissue three weeks after AMI (Figure 7). However, there were no labeled cells found 10 weeks after AMI.

\section{Discussion}

A variety of cell types from different sources have been proposed for cell-based cardiac repair. However, the findings of previous studies have been mixed [1], and the underlying mechanism of cell-based therapy is not well understood. In addition, the ideal cell type still needs to be determined, and the delivery strategy needs to be further optimized. In this study, we compared the two most clinically relevant cell types for the treatment of AMI. With a representative preclinical porcine model, we found that percutaneous intramyocardial delivery of MSC induced superior improvement of regional left ventricular function compared with BMMNC. Treatment with MSC increased vascular density and decreased CVF.

An effective cellular delivery method is a prerequisite for successful stem cell therapy. To date, most clinical trials have utilized intracoronary delivery. However, there are many studies showing that intracoronary cell injections of MSC could cause no-reflow or micro-infarctions [19]. Intravenous injection has been shown to be an ineffective delivery approach with insufficient engrafted cells homing into the myocardium [20]. Freyman et al. demonstrated that intramyocardial delivery may be more effective than intracoronary and intravenous delivery [14]. Other studies have shown that intramyocardial delivery can be safely used in ischemic cardiomyopathy patients [15]. Therefore, in the present study, we used a percutaneous intramyocardial injection to deliver cells through a cardiac catheter, mimicking the clinical scenario. In our study, although transient ventricular arrhythmias occurred when the injection catheter contacted the myocardium, there were noserious adverse events, which demonstrated the safety of in- 
tramyocardial delivery.

In the early stages after MI, stem cell survival may be compromised by inflammation, microvascular obstruction, and reperfusion injury, while at later stages, stem cell homing may be compromised by scar formation and cytokine down regulation[21]. Although the ideal time point for transplantation remains to be defined, it is most likely between two and 14 days after the onset of AMI[22]. In our study, we chose to perform cell transplantation two weeks after AMI instead of simultaneously with AMI for the following reasons. First, two weeks post-AMI, stem cell homing could be less affected by damage to the micro environment such as inflammation, microvascular obstruction, scar formation, or cytokine down regulation. Second, it is possible that cell injection at the time of induced AMI could cause higher mortality in minipigs during the experimental procedure.

In the present study, we observed a superior protective effect of MSC compared with BMMNC. Treatment with MSC induced better improvement in regional left ventricular function, which was associated with increased vascular density and decreased CVF. Similarly, Mazo et al. carried out a comparative study performed on a rat chronic myocardial infarction model and found a better long-term effect from MSC than BMMNC treatment [23]. However, Vander Bogt et al. reported a better functional improvement following BMMNC transplantation compared with MSC transplantation in a mouse AMI model [24]. Several reasons could account for these distinct results, including different experimental animals, cell doses, and delivery time points. For example, Mazo et al. injected cells five weeks after AMI, whereas Van der Bogt et al. injected cells immediately after AMI. In our study, we performed cell transplantation two weeks after AMI. Cell dose could also explain the different experimental results. However, a recent study found that direct MSC delivery into infarcted myocardium showed no dose-dependent effect. MSC injection exerted therapeutic effects of decreasing cardiac infarct size by affecting the viability of native cardiomyocytes through an undefined signaling mechanism [25].

The therapeutic effects of cell therapy for MI remain controversial. Some experiments and clinical trials have shown negative effects of stem cell treatment on LVEF $[4,26]$. We observed regional left ventricular function improvement without LVEF recovery in the cell therapy groups in the present study. The reason for this finding might be that the small number of engrafted cells may not have been sufficient to reverse the moderate infarction size and to induce significant improvement in global left ventricular function.
In the present study, we observed transient survival of engrafted bone marrow cells in the host heart three weeks after AMI, but the transplanted cells could hardly be detected 10 weeks after AMI. This result is consistent with the findings of several recent studies $[27,28]$. It seems that cells can only persist for a limited time in ischemic host cardiac tissue. However, in the present study, improved myocardial perfusion and increased capillary density were found in both cell-treated groups. Therefore, we hypothesized that an indirect paracrine effect could account for this finding. It has been reported that MSC secrete different growth factors than BMMNC, which might explain the different effects of MSC and BMMNC [29].

It has been shown that myocardial fibrosis is the replacement of cardiomyocytes by collagen and that matrix metallo proteinases (MMP) and tissue inhibitors of metallo proteinases (TIMP) are involved [30]. Evidence suggests that the injection of mesenchymal precursor cells could alter collagen dynamics, MMP, and TIMP levels in a sheep MI modeland that MSC transplantation improves ventricular function in a rat model of dilated cardiomyopathy (DCM), partly through inhibition of myocardial fibrosis. In our study, we found that MSC decreased myocardial fibrosis in a minipig MI model, implying that MSC might have beneficial paracrine effects on myocardial fibrosis.

There are some limitations to the present study. One is the small sample size. Second, we did not fully assess the impact of accompanied pharmaco-therapy post-AMI in this animal study. In a clinical setting, it is possible that pharmaco therapy could influence the magnitude of therapeutic responses[31].In addition, long-term in vivo cell monitoring in large living animals is difficult and more sensitive and specific molecular imaging techniques are needed. Finally, we only studied a single dose of BMMNC and MSC, and the ideal cell dose is yet to be determined.

In conclusion, the present study indicated that the percutaneous intramyocardial delivery of both BMMNC and MSC produces beneficial effects on perfusion in an infarcted heart as determined by SPECT/CT imaging. Furthermore, the injection of MSC produced superior improvement in regional cardiac function improvement compared with BMMNC, partly through an indirect paracrine effect.

\section{Acknowledgements}

This work was supported by the National Distinguished Young Scientists Grant of China (81325009) and the National Nature Science Foundation of China (No.81270168, No.81090274, No.81227901, No.81130072, No.81230033, and No.81030002), (FCao BWS12J037), an Innovation 
Team Development Grant by the China Department of Education (IRT1053), and the National Basic Research Program of China (2012CB518101).

We would like to thank Microport, Inc. for kindly providing the percutaneous intramyocardial injection catheter system. We also wish to thank Xinxing Song and Tong Liu for helping to establish the porcine MI model.

\section{Abbreviations}

AMI: acute myocardial infarction; BMMNC: bone marrow-derived mononuclear cells; MSC: mesenchymal stem cells; LAD: left anterior descending; SPECT/CT: gated single-photon emission computed tomography/computed tomography; LVEF: Left ventricular ejection fraction; LVESV: Left ventricular end-systolic volume; LVEDV: Left ventricular end-diastolic volumes; WMSI: wall motion score index.

\section{Competing Interests}

The authors have declared that no competing interest exists.

\section{References}

1. Rosenzweig A. Cardiac cell therapy--mixed results from mixed cells. N Engl J Med. 2006; 355: 1274-7. doi:10.1056/NEJMe068172.

2. Wollert KC, Meyer GP, Lotz J, Ringes-Lichtenberg S, Lippolt P, Breidenbach C, et al. Intracoronary autologous bone-marrow cell transfer after myocardial infarction: the BOOST randomised controlled clinical trial. Lancet. 2004; 364: 141-8. doi:10.1016/S0140-6736(04)16626-9.

3. Assmus B, Honold J, Schachinger V, Britten MB, Fischer-Rasokat U, Lehmann $\mathrm{R}$, et al. Transcoronary transplantation of progenitor cells after myocardial infarction. N Engl J Med. 2006; 355: 1222-32. doi:10.1056/NEJMoa051779.

4. Janssens S, Dubois C, Bogaert J, Theunissen K, Deroose C, Desmet W, et al. Autologous bone marrow-derived stem-cell transfer in patients with ST-segment elevation myocardial infarction: double-blind, randomised controlled trial. Lancet. 2006; 367: 113-21. doi:10.1016/S0140-6736(05)67861-0.

5. Lunde K, Solheim S, Aakhus S, Arnesen H, Abdelnoor M, Egeland T, et al. Intracoronary injection of mononuclear bone marrow cells in acute myocardial infarction. N Engl J Med. 2006; 355: 1199-209. doi:10.1056/NEJMoa055706.

6. Schachinger V, Erbs S, Elsasser A, Haberbosch W, Hambrecht R, Holschermann $\mathrm{H}$, et al. Intracoronary bone marrow-derived progenitor cells in acute myocardial infarction. N Engl J Med. 2006; 355: 1210-21. doi:10.1056/NEJMoa060186.

7. Cao F, Sun D, Li C, Narsinh K, Zhao L, Li X, et al. Long-term myocardial functional improvement after autologous bone marrow mononuclear cells transplantation in patients with ST-segment elevation myocardial infarction: 4 years follow-up. Eur Heart J. 2009; 30: 1986-94. doi:10.1093/eurheartj/ehp220.

8. Pittenger MF, Mackay AM, Beck SC, Jaiswal RK, Douglas R, Mosca JD, et al. Multilineage potential of adult human mesenchymal stem cells. Science. 1999; 284: 143-7.

9. Amado LC, Saliaris AP, Schuleri KH, St John M, Xie JS, Cattaneo S, et al. Cardiac repair with intramyocardial injection of allogeneic mesenchymal stem cells after myocardial infarction. Proc Natl Acad Sci U S A. 2005; 102: 11474-9. doi:10.1073/pnas.0504388102.

10. Halkos ME, Zhao ZQ, Kerendi F, Wang NP, Jiang R, Schmarkey LS, et al. Intravenous infusion of mesenchymal stem cells enhances regional perfusion and improves ventricular function in a porcine model of myocardial infarction. Basic Res Cardiol. 2008; 103: 525-36. doi:10.1007/s00395-008-0741-0.

11. Quevedo HC, Hatzistergos KE, Oskouei BN, Feigenbaum GS, Rodriguez JE, Valdes D, et al. Allogeneic mesenchymal stem cells restore cardiac function in chronic ischemic cardiomyopathy via trilineage differentiating capacity. Proc Natl Acad Sci U S A. 2009; 106: 14022-7. doi:10.1073/pnas.0903201106.

12. Parekkadan B, Milwid JM. Mesenchymal stem cells as therapeutics. Annu Rev Biomed Eng. 2010; 12: 87-117. doi:10.1146/annurev-bioeng-070909-105309.

13. Niccoli-Asabella A, Ferlan G, Crovace A, Notaristefano A, Rubini D, Altini C, et al. Swine experimental model to evaluate stem cells implant post myocardial infarction by perfusion gated-SPET. Hell J Nucl Med. 2012; 15: 16-22.

14. Freyman T, Polin G, Osman H, Crary J, Lu M, Cheng L, et al. A quantitative, randomized study evaluating three methods of mesenchymal stem cell deliv- ery following myocardial infarction. Eur Heart J. 2006; 27: 1114-22. doi:ehi818 [pii]10.1093/eurheartj/ehi818.

15. Williams AR, Trachtenberg B, Velazquez DL, McNiece I, Altman P, Rouy D, et al. Intramyocardial stem cell injection in patients with ischemic cardiomyopathy: functional recovery and reverse remodeling. Circ Res. 2011; 108: 792-6. doi:10.1161/CIRCRESAHA.111.242610.

16. Hatzistergos KE, Quevedo H, Oskouei BN, Hu Q, Feigenbaum GS, Margitich IS, et al. Bone marrow mesenchymal stem cells stimulate cardiac stem cell proliferation and differentiation. Circ Res. 2010; 107: 913-22. doi:10.1161/CIRCRESAHA.110.222703.

17. Kim BO, Tian H, Prasongsukarn K, Wu J, Angoulvant D, Wnendt S, et al. Cell transplantation improves ventricular function after a myocardial infarction: a preclinical study of human unrestricted somatic stem cells in a porcine model. 2005 doi:10.1161/01.CIRCULATIONAHA.105.524678.

18. Gavira JJ, Nasarre E, Abizanda G, Perez-Ilzarbe M, de Martino-Rodriguez A, Garcia de Jalon JA, et al. Repeated implantation of skeletal myoblast in a swine model of chronic myocardial infarction. Eur Heart J. 2010; 31: 1013-21. doi:ehp342 [pii]10.1093/eurheartj/ehp342.

19. Vulliet PR, Greeley M, Halloran SM, MacDonald KA, Kittleson MD. Intra-coronary arterial injection of mesenchymal stromal cells and microinfarction in dogs. Lancet. 2004; 363: 783-4. doi:10.1016/S0140-6736(04)15695-X.

20. Hofmann M, Wollert KC, Meyer GP, Menke A, Arseniev L, Hertenstein B, et al. Monitoring of bone marrow cell homing into the infarcted human myocardium. Circulation. 2005; 111: 2198-202. doi: 10.1161/01.CIR.0000163546.27639.AA.

21. Schoenhard JA, Hatzopoulos AK. Time is like a clock in my heart: implications for stem cell delivery after myocardial infarction. Cardiology. 2010; 117: 158-60. doi:10.1159/000321396.

22. Strauer BE, Steinhoff G. 10 years of intracoronary and intramyocardial bone marrow stem cell therapy of the heart: from the methodological origin to clinical practice. J Am Coll Cardiol. 2011; 58: 1095-104. doi:S0735-1097(11)02285-6 [pii]10.1016/j.jacc.2011.06.016.

23. Mazo M, Gavira JJ, Abizanda G, Moreno C, Ecay M, Soriano M, et al. Transplantation of mesenchymal stem cells exerts a greater long-term effect than bone marrow mononuclear cells in a chronic myocardial infarction model in rat. Cell Transplant. 2010; 19: 313-28. doi:10.3727/096368909X480323.

24. van der Bogt KE, Sheikh AY, Schrepfer S, Hoyt G, Cao F, Ransohoff KJ, et al. Comparison of different adult stem cell types for treatment of myocardial is$\begin{array}{llll}\text { chemia. } & \text { Circulation. }\end{array}$ doi:10.1161/CIRCULATIONAHA.107.759480.

25. Hashemi SM, Ghods S, Kolodgie FD, Parcham-Azad K, Keane M, Hamamdzic $\mathrm{D}$, et al. A placebo controlled, dose-ranging, safety study of allogenic mesenchymal stem cells injected by endomyocardial delivery after an acute myocardial infarction. Eur Heart J. 2008; 29: 251-9. doi:10.1093/eurheartj/ehm559.

26. Moelker AD, Baks T, van den Bos EJ, van Geuns RJ, de Feyter PJ, Duncker DJ, et al. Reduction in infarct size, but no functional improvement after bone marrow cell administration in a porcine model of reperfused myocardial infarction. Eur Heart J. 2006; 27: 3057-64. doi:10.1093/eurheartj/ehl401.

27. Jameel MN, Li Q, Mansoor A, Qiang X, Sarver A, Wang X, et al. Long-term functional improvement and gene expression changes after bone marrow-derived multipotent progenitor cell transplantation in myocardial infarction. Am J Physiol Heart Circ Physiol. 2010; 298: H1348-56. doi:10.1152/ajpheart.01100.2009.

28. Sheikh AY, Huber BC, Narsinh $\mathrm{KH}$, Spin JM, van der Bogt $\mathrm{K}$, de Almeida PE, et al. In vivo functional and transcriptional profiling of bone marrow stem cells after transplantation into ischemic myocardium. Arterioscler Thromb Vasc Biol. 2012; 32: 92-102. doi:10.1161/ATVBAHA.111.238618.

29. Li TS, Cheng K, Malliaras K, Smith RR, Zhang Y, Sun B, et al. Direct comparison of different stem cell types and subpopulations reveals superior paracrine potency and myocardial repair efficacy with cardiosphere-derived cells. J Am Coll Cardiol. 2012; 59: 942-53. doi:10.1016/j.jacc.2011.11.029.

30. Ohnishi S, Sumiyoshi H, Kitamura S, Nagaya N. Mesenchymal stem cells attenuate cardiac fibroblast proliferation and collagen synthesis through paracrine actions. FEBS Lett. 2007; 581: 3961-6. doi:10.1016/j.febslet.2007.07.028.

31. Yang YJ, Qian HY, Huang J, Geng YJ, Gao RL, Dou KF, et al. Atorvastatin treatment improves survival and effects of implanted mesenchymal stem cells in post-infarct swine hearts. Eur Heart J. 2008; 29: 1578-90. doi:10.1093/eurheartj/ehn167. 